\title{
ARTICLE
}

Translational Therapeutics

\section{CRISPR/Cas9 genome-wide loss-of-function screening identifies druggable cellular factors involved in sunitinib resistance in renal cell carcinoma}

\author{
Peter Makhov (D) ${ }^{1}$, Ji A. Sohn², llya G. Serebriiskii ${ }^{1,3}$, Rushaniya Fazliyeva ${ }^{4}$, Vladimir Khazak ${ }^{5}$, Yanis Boumber ${ }^{6}$, Robert G. Uzzo $^{7}$ and $^{2}$ \\ Vladimir M. Kolenko ${ }^{4}$
}

BACKGROUND: Multi-targeted tyrosine kinase inhibitors (TKIs) are the standard of care for patients with advanced clear cell renal cell carcinoma (ccRCC). However, a significant number of ccRCC patients are primarily refractory to targeted therapeutics, showing neither disease stabilisation nor clinical benefits.

METHODS: We used CRISPR/Cas9-based high-throughput loss of function (LOF) screening to identify cellular factors involved in the resistance to sunitinib. Next, we validated druggable molecular factors that are synthetically lethal with sunitinib treatment using cell and animal models of cCRCC.

RESULTS: Our screening identified farnesyltransferase among the top hits contributing to sunitinib resistance in ccRCC. Combined treatment with farnesyltransferase inhibitor lonafarnib potently augmented the anti-tumour efficacy of sunitinib both in vitro and in vivo.

CONCLUSION: CRISPR/Cas9 LOF screening presents a promising approach to identify and target cellular factors involved in the resistance to anti-cancer therapeutics.

British Journal of Cancer (2020) 123:1749-1756; https://doi.org/10.1038/s41416-020-01087-x

\section{BACKGROUND}

Renal cell carcinoma (RCC) is the most common type of kidney cancer with rising incidence. ${ }^{1}$ It is categorised into various subtypes, with clear cell RCC (ccRCC) representing $\sim 85 \%$ of all RCC tumours. ${ }^{2}$ Papillary RCC and chromophobe RCC represent the most common remaining histologic subtypes with an incidence of $7-14 \%$ and $6-11 \%$, respectively. ${ }^{2}$ Current targeted molecular strategies, including multitargeted tyrosine kinase inhibitors (TKIs), have resulted in a doubling of progression-free survival and significant gains in overall survival, thereby notably changing the treatment paradigm of advanced kidney cancer. ${ }^{3,4}$ Yet, about onequarter of the cCRCC patients are primarily refractory to treatment with TKIs. ${ }^{5}$ Furthermore, most patients that respond initially will typically progress within 12 months of starting therapy. ${ }^{6}$

The ability of sunitinib to inhibit angiogenesis is wellestablished. However, we and others have demonstrated that, at concentrations found in human tumour specimens, ${ }^{7}$ sunitinib may also manifest a direct suppressive effect on tumour cells of various origins. ${ }^{8-11}$ Studies by Hillman et al. indicate that sunitinib exerts a direct cytotoxic effect on RCC cells at doses $>0.5 \mu \mathrm{M} .{ }^{12}$ Concentrations of sunitinib in human tumour specimens can reach $9.5+$ $2.4 \mu \mathrm{mol} / \mathrm{L}$, whereas plasma concentrations were found to be significantly lower, $0.3+0.1 \mu \mathrm{mol} / \mathrm{L} .{ }^{11}$ These findings suggest that sunitinib may selectively accumulate in tumour tissue at high concentrations and exert direct cytotoxic effect on tumour cells. Furthermore, some clinical studies suggest that the high response rate may result from a direct effect of TKIs on malignant cells. ${ }^{13}$

Protein farnesylation, catalysed by protein farnesyltransferase (FTase), plays important roles in the membrane association and protein-protein interaction of a number of eukaryotic proteins. ${ }^{14}$ FTase is located in the cell cytosol, and it is one of the three enzymes in the prenyltransferase group that catalyses most isoprenylation reactions. FTase adds a 15 -carbon isoprenoid lipid (the farnesyl group) to proteins bearing a CAAX motif. ${ }^{15}$ Preclinical studies in the 1990s demonstrated that FTase inhibitors (FTIs) could successfully kill cancer cells both in vitro and in vivo with very little toxicity, thus generating much excitement toward the development of FTls-based anti-cancer therapeutic regimens. ${ }^{16}$ Unfortunately, in most clinical trials, FTIs as monotherapy have not been as successful as expected. ${ }^{16-19}$ However, a combination of FTls with cytotoxic agents improved the responses of patients with locally advanced breast cancer and some other advanced solid tumours. ${ }^{16,20-22}$

Synthetic lethality screens hold great promise for the development of novel therapeutic interventions. We have applied CRISPR/ Cas9-based high-throughput loss-of-function (LOF) screening to

\footnotetext{
${ }^{1}$ Molecular Therapeutics Program, Fox Chase Cancer Center, Philadelphia, PA, USA; ${ }^{2}$ Blood Cell Development and Function Program, Fox Chase Cancer Center, Philadelphia, PA,

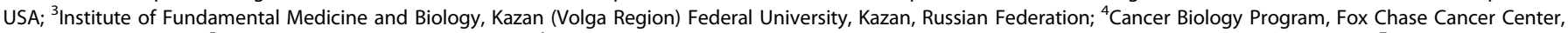

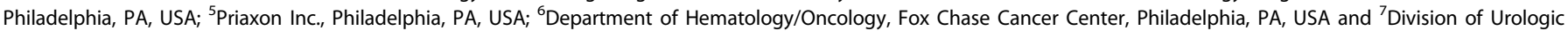
Oncology, Department of Surgery, Fox Chase Cancer Center, Philadelphia, PA, USA Correspondence: Peter Makhov (Petr.Makhov@fccc.edu)
} 
identify genes involved in the resistance to sunitinib, a standard front-line therapeutic agent for the treatment of advanced ccRCC. Our search identified FTase and its downstream effectors among the top hits. Treatment of the sunitinib-resistant $786-O$ and PNX0010 ccRCC cells ${ }^{9,23}$ with FTI lonafarnib potently augmented the in vitro anti-tumour efficacy of sunitinib. Moreover, combined treatment with lonafarnib circumvented resistance to sunitinib in the PNX0010 xenograft tumour model. Therefore, concomitant treatment with lonafarnib and sunitinib may represent a rational therapeutic strategy for $\mathrm{ccRCC}$ patients with sunitinib-resistant tumours.

\section{METHODS}

Cells and culture conditions

The 786-O human RCC cell line was obtained from ATCC. PNX0010 ccRCC cell line, which was described previously, ${ }^{10,24}$ was established from a fresh tumour specimen obtained intraoperatively from an RCC patient, undergoing nephron-sparing surgery at Fox Chase Cancer Center. This cell population is clinically correlated to an aggressive variant of ccRCC. PNX0010 cells are VHL-negative and express SETD2, BAP1 and PBRM1 proteins (Supplementary Fig. S1). Initial stocks were cryopreserved, and at every 6-month interval a fresh aliquot of frozen cells was used for the experiments. No authentication was done by the authors. Cells were cultured in RPMI 1640 (Bio-Whittaker) supplemented with $10 \%$ FCS (Hyclone), gentamicin $(50 \mathrm{mg} / \mathrm{l})$, sodium pyruvate $(1 \mathrm{mM})$ and non-essential amino acids $(0.1 \mathrm{mM})$ under conditions indicated in the figure legends.

Antibodies and reagents

Sunitinib (\#13159) and lonafarnib (\#11746) were obtained from Cayman Chemical Company (Ann Arbor, MI). Anti-FNTB (\#ab109625) antibody was obtained from Abcam (Cambridge, UK). Anti- $\beta$-actin (\#3700), SETD2 (23486) and anti-PBRM1 (91894) antibodies were obtained from Cell Signaling Technology, (Danvers, MA). Anti-BAP1(sc-28383) antibody was obtained from Santa Cruz (Dallas, TX).

\section{Generation of sgRNA library}

The custom oligonucleotide array (Supplementary Table 1) was synthesised by Custom Array Inc. Overlapping PCR was performed to incorporate Ndel and Xbal sites to the custom array for subsequent Gibson Assembly (NEB, Ipswich, MA). The PCR products were then cloned into pLX-sgRNA linearised with Ndel and Xbal. pLX-sgRNA was a kind gift from Eric Lander \& David Sabatini (Addgene plasmid \#50662). ${ }^{25}$ The Gibson library reaction was transformed into XL10-Ultra competent cells. To maintain the complexity of the library, at least 20 -fold coverage in library representation was recovered in the transformation and cultured in NYZM + broth for $7 \mathrm{~h}$ or until OD600 reached 0.8 . Subsequently, deep sequencing (Illumina) was performed to validate the library complexity of the input plasmid and lentivirus pool.

\section{CRISPR/Cas9-based genome-wide LOF screening}

The RNA-guided CRISPR-associated nuclease Cas9 provides an effective means of introducing targeted LOF mutations at specific sites in the genome. ${ }^{26}$ For lentiviral production, we used 293T cells transfected with pCW-Cas9 encoding FLAG-tagged Cas9 nuclease driven by doxycycline-inducible promoter and carrying puromycin resistance marker. 786-O ccRCC cells ${ }^{9,27}$ were infected with Cas9 expressing lentivirus at high multiplicity of infection (MOI) and the stable clones were selected using puromycin. Cas9 induction in the individual clones was assessed by immunoblotting for Cas9 protein. A clone with superior Cas9 induction rate was selected and the cells were subjected to the infection with human CRISPR sgRNA library (containing blasticidin resistance marker) targeting 18,000 genes with 90,000 individual sgRNAs ( 5 sgRNA per gene) at low $\mathrm{MOI}<1$. The infected cells were selected using blasticidin and frozen for future manipulations. Deep sequencing on an Illumina Nextseq was used to monitor library composition. Trimmed sequences were aligned to libraries using Bowtie, with zero mismatches tolerated. All alignments from multi-mapped reads were used. Enrichment of individual hairpins was calculated as a median-normalised log-ratio of the fraction of counts. We assigned a four-fold reduction in sgRNAs abundance as significant. A previously established gold standard of 217 genes expected to have growth phenotypes in all cell types (essential) and 947 genes expected to have growth phenotypes in no cell type (nonessential) was used to estimate true positive and false-positive rates. We calculated the number of sgRNAs with significant abundance decrease for all essential and non-essential genes present in our library. False-negative rate was calculated as the ratio of the number of essential genes, for which no decrease in abundance was detected during induction, to the total number of essential genes present in our library. False-positive rate was calculated as the ratio of the number of non-essential genes with a significant decrease in abundance during induction, to the total number of non- essential genes present in our library. We have used the cutoff (no less than 4 sgRNA with no less than 4 -fold reduction in abundance) to stratify the candidate list and identify genes contributing to sunitinib resistance.

\section{Western blot analysis}

Cell lysates preparation and western blot analysis were performed as described previously. ${ }^{28}$

Cell viability and drug interaction analysis

Cell viability was analysed by CellTiter Blue cell viability assay (\#G8081) (Promega) as described previously. ${ }^{10}$ Effective doses (EDs) were calculated using XLfit, a Microsoft Excel add-in. The synergistic interaction between sunitinib and lonafarnib was evaluated by the combination index $(\mathrm{Cl})$ using CalcuSyn 2.0 software. ${ }^{29} \mathrm{Cl}<0.1$ very strong synergism; $0.1-0.3$ strong synergism; 0.3-0.7 synergism; $0.7-0.9$ moderate to slight synergism; $0.9-1.1$ nearly additive; 1.1-1.45 slight to moderate antagonism; $1.45-3.3$ antagonism; $>3.3$ strong to very strong antagonism.

Analysis of apoptosis

DNA fragmentation was detected using APO-BRDU kit (\#AU1001) (The Phoenix Flow Systems, Inc., San Diego, CA).

Lysosomal Sunitinib sequestration analysis

786-O cells were plated into 96 -well plate $\left(3 \times 10^{3}\right.$ cells per well). Next day, cells were pre-incubated with Lonafarnib $(10 \mu \mathrm{M})$ for $8 \mathrm{~h}$. Sunitinib $(10 \mu \mathrm{M})$ were then added to wells with, or without lonafarnib and incubation continued for next $24 \mathrm{~h}$. Image acquisition was performed on an ImageXpress micro automated imaging system (Molecular Devices, Sunnyvale, CA) driven by MetaXpress software. Nine image fields per well were acquired, using three channels to capture matching signal from Hoechststained nuclei (DAPI channel, ex 377/50, em 447/60), and vesicles in both fluorescein (for sunitinib) and TRITC (for LysoTracker Red) wavelengths (ex 472/30 em 520/35; ex 525/40, em 585/40 respectively). Epifluorescence images were acquired with a $20 \mathrm{x}$ objective (ELWD Plan Fluor, NA 0.45, WD 7.4), using laser autofocus with a z-offset. Images were analysed using 'Multiwavelength Scoring' MetaXpress module for measurement of parameters within each fluorescent channel. Data generated from these analyses were displayed within Acuity Xpress (Molecular Devices).

\section{siRNA transfection}

786-O and PNX0010 cells were transfected with pooled siRNA mix (Cat\# SI00031717 and SI00031731) targeting Protein Farnesyltransferase subunit $\beta$ (FNTB), or non-silencing siRNA (Cat\# 
0001027281) (Qiagen, Frederick, MD) using Lipofectamine RNAiMAX Transfection Reagent (Thermo Fisher Scientific Inc., Waltham, MA) according to the manufacturer's instructions. In all, $48 \mathrm{~h}$ posttransfection the efficacy of knockdown was validated by Western blotting analysis using specific antibodies.

Generation of cell lines with deleted CAAX-motifs of Rheb, Rab7a and Rab51

Mutant $786-0$ cells were generated using pLenti-CRISPRv2 lentiviral vectors expressing sgRNAs targeting C-end of Rheb, Rab7a and Rab25. Targeting sequences are as follows: 5'- ggagg cagaaaaaatggacg (Rheb); 5'- ggacaagaatgaccgggcca (Rab7a) and 5 '-gcccaggctggacaggagcc (Rab25). Lentiviral production and infection of 786-O cells were performed as described above. After the selection with puromycin $(1 \mu \mathrm{g} / \mathrm{ml})$, cells were grown at least for 10-14 days and genomic DNA was isolated using Quick-DNA Miniprep kit (Zymo-Research, Irvine, CA) The amplicons spanning the sgRNAs targeting sites were generated by PCR using Ex Taq DNA polymerase, hot-start version (Takara Bio USA, Inc., Mountain View, CA) and specific primers (Supplementary Table S1). The efficacy of knockouts was validated by direct sequencing of amplicons (Supplementary Fig. S2).

Assessment of in vivo tumour growth

For in vivo studies, $1 \times 106$ of PNX0010 cells were inoculated s.c. in the flank region of 6-week-old male C.B17/lcr-scid mice (all animal procedures were done in accordance with institutional guidelines on animal care and with appropriate institutional certification; IACUC protocol \#13-16). Animals were fed an autoclaved 2018SX diet (Harlan Teklad, Madison, WI) and water ad libitum. Two weeks after the injection of tumour cells, animals were randomly assigned to the control or experimental groups. The sample size $n=5$ mice/group was selected because the effects of concomitant treatment with sunitinib and linafarnib were evaluated in vivo for the first time in the present study. The mice were treated orally three times per week with: (i) $10 \%$ 2-hydroxypropyl- $\beta$-cyclodextrin in PBS (vehicle); (ii) sunitinib (40 mg/kg); (iii) lonafarnib $(40 \mathrm{mg} / \mathrm{kg}$ ); (iv) sunitinib $(40 \mathrm{mg} / \mathrm{kg})$ and lonafarnib $(40 \mathrm{mg} / \mathrm{kg})$ combined. Tumour volumes were calculated using the formula: (volume $=$ $0.52 \times(\text { width })^{2} \times$ length) as described previously. ${ }^{23}$ The mice were euthanised using slow introduction of $\mathrm{CO}_{2}$ into the chamber. The flow rate for $\mathrm{CO}_{2}$ was set to $10-30 \%$ displacement of the chamber volume/min.

Statistical analysis

Statistical analysis was performed using a two-sided Student's $t$-test. A $p$-value of $<0.05$ was considered statistically significant.

\section{RESULTS}

CRISPR/Cas9-based genome-wide LOF screening to identify cellular factors contributing to sunitinib resistance in cCRCC To identify cellular factors involved in sunitinib resistance, we infected 786-O ccRCC cells with human CRISPR sgRNA library as described in Methods. A brief overview of this strategy is depicted in Fig. 1. Next, we have characterised our library-transformed 786$O$ cell line and optimised the hit selection parameters using a previously established gold standard set of essential and nonessential genes. ${ }^{30}$ It is expected that after the induction, most of cells harbouring the essential genes knockout should be eliminated, while the non-essential genes should be largely retained. A set of 216 essential genes and 771 nonessential genes present in our library was used to estimate true positive and falsepositive rates. By targeting the false-positive rate to be below $1 \%$, we have established the cut-off of no less than 4 sgRNA per gene, with no less than 4-fold reduction in abundance as the threshold for significance (Table 1). Using these stringent criteria, we were able to recover only $\sim 12 \%$ of true positives, but this stratification

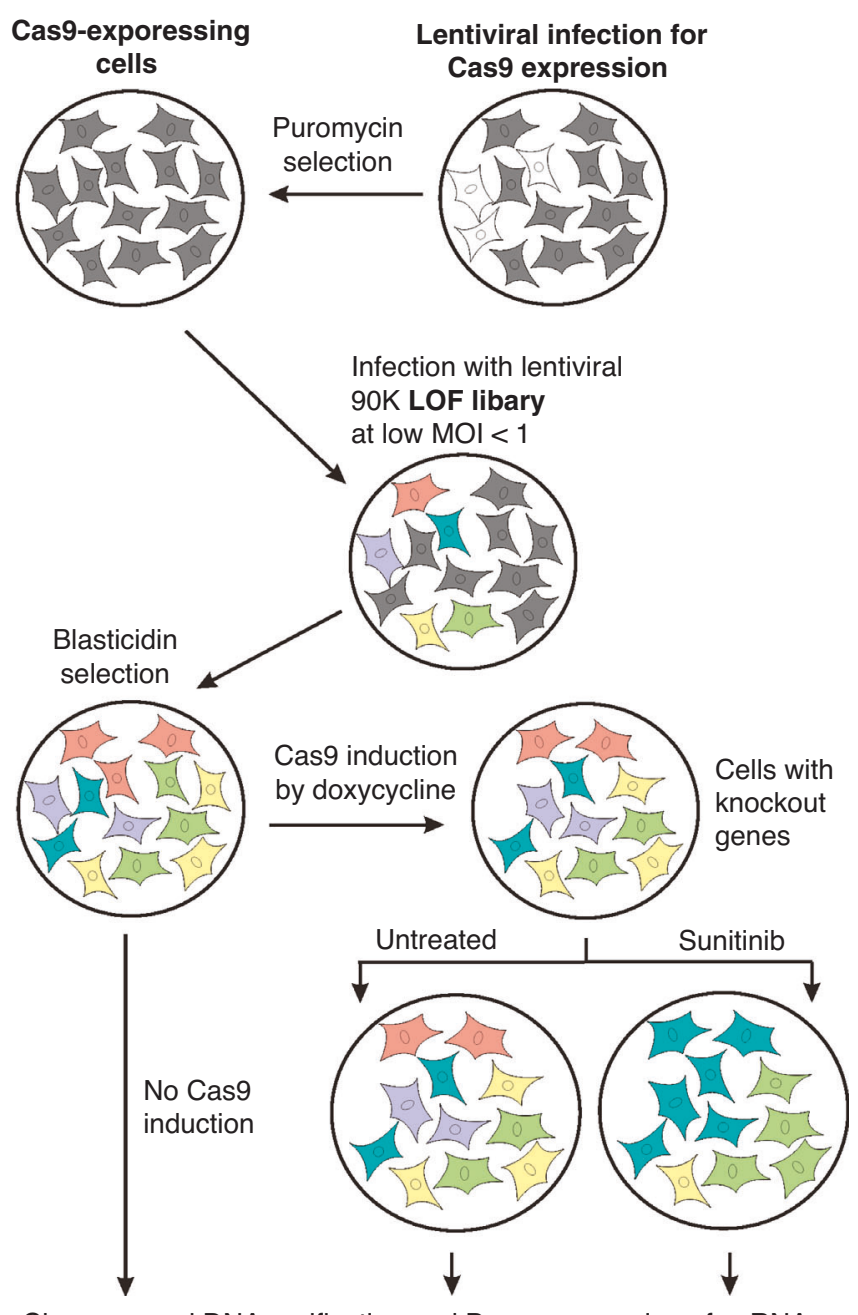

Chromosomal DNA purification and Deep sequencing of sgRNAs Identify sgRNAs underrepresented in the surviving cell population

Fig. 1 Chart depicting CRISPR/Cas9-based LOF screening strategy to identify molecular targets synthetically lethal with sunitinib treatment.

Table 1. The cutoff criteria for evaluation of true positive and falsepositive rates.

\begin{tabular}{llllll}
\hline & \multicolumn{5}{c}{$\begin{array}{l}\text { Cutoff (number of sgRNAs with } \\
\text { 4-fold reduction) }\end{array}$} \\
\cline { 3 - 7 } & Total & $>1$ & $>2$ & $>3$ & $>4$ \\
\hline Essential genes & 216 & 138 & 67 & 25 & 5 \\
Non-essential genes & 771 & 105 & 15 & 2 & 0 \\
False-negative rate: & & $36.1 \%$ & $69.0 \%$ & $88.4 \%$ & $97.7 \%$ \\
False-positive rate: & & $13.6 \%$ & $1.9 \%$ & $0.3 \%$ & $0.0 \%$ \\
\hline
\end{tabular}

allowed us to optimise the subsequent validation by concentrating on the most reliable candidates first.

Next, 786-O cells were incubated with sunitinib at $10 \mu \mathrm{M}$ for 12 days (about six passages). This concentration represents the intratumoural concentration of sunitinib in human tumour specimens $(9.5 \pm 2.4 \mu \mathrm{mol} / \mathrm{L}){ }^{11}$ We anticipated that during this time the cells with those knockout genes, which contribute to the resistance to sunitinib would be eliminated from the population. Next, we identified underrepresented sgRNAs and their 
corresponding gene targets in the surviving cell population. The primers corresponding to sequences flanking the guide in the lentiviral vector included 8-bp bar codes for Illumina-based sequencing. Thus, each sgRNA served as an individual DNA barcode that was used to count the number of cells carrying guides by sequencing. Our search identified a number of genes potentially involved in sunitinib resistance in ccRCC (Supplementary Table S2). ${ }^{23}$

Identification and validation of druggable molecular factors that are synthetically lethal with sunitinib treatment

Based on the highest rank of identified hits, we have chosen to focus on the genes, which have not been previously reported to be involved in sunitinib resistance. Our screen identified farnesyltransferase among the top hits. Farnesyltransferase acts as a hetero-dimer comprising from $a$ and $\beta$ subunits (encoding by genes FNTA and FNTB). To validate whether farnesyltransferase plays a significant role in sunitinib resistance, we performed siRNA mediated depletion of $\beta$ subunit of this enzyme in 786-O and PNX0010 cells (Fig. 2a). After $24 \mathrm{~h}$, siRNA transfected cells were treated with $10 \mu \mathrm{M}$ of sunitinib for the next $48 \mathrm{~h}$. As demonstrated in Fig. 2b, the knockdown of farnesyltransferase has dramatically sensitised ccRCC cells to sunitinib-mediated apoptosis.

The search through clinicaltrials.gov identified several pharmacological agents inhibiting farnesyltransferase function, including lonafarnib. ${ }^{31,32}$ Viability of $786-O$ and PNX0010 cell subjected to treatment with lonafarnib and sunitinib was examined using
CellTiter Blue viability assay. The effective doses (EDs) of both drugs were assessed using XLfit (Fig. 3a). Next, 786-O and PNX0010 cell were treated with various dosing regimens of lonafarnib and sunitinib to examine a synergistic anti-tumour effect for the combination of these agents. The data analysis using CalcuSyn 2.0 software revealed a high level of synergistic interaction between lonafarnib and sunitinib (Fig. 3b, c). Concomitant treatment with lonafarnib and sunitinib at higher doses resulted in profound DNA fragmentation in 786-O and PNX0010 cells (Fig. 3d).

Lysosomal sequestration of sunitinib limits its intracellular antitumour activity. ${ }^{11}$ To further define the mechanism of lonafarnibmediated sensitisation of tumour cells to sunitinib, we examined whether the lysosomal sequestration of sunitinib can be disrupted by lonafarnib. Sunitinib is a fluorescent compound. Therefore, its intracellular uptake and localisation can easily be monitored. As demonstrated in Fig. 4a, b, treatment with lonafarnib significantly reduced a number of sunitinib-containing lysosomes. Importantly, the total cellular accumulation of sunitinib was not affected by lonafarnib (Fig. 4c).

Our recent studies demonstrate that co-administration of mTORC1 inhibitors overcomes sunitinib resistance in renal and prostate cancer cells both in vitro and in vivo. ${ }^{9}$ mTORC1 is activated through the direct binding of Rheb, ${ }^{33}$ a GTPase upregulated in transformed cells. ${ }^{34}$ The farnesylation of Rheb is required for its lysosomal membrane localisation and activation of mTORC1 signalling. ${ }^{35,36}$ FTIs suppress Rheb farnesylation and

a

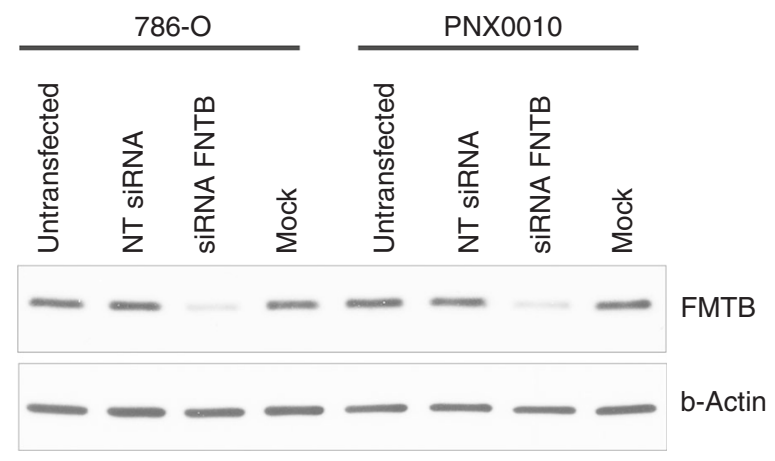

b
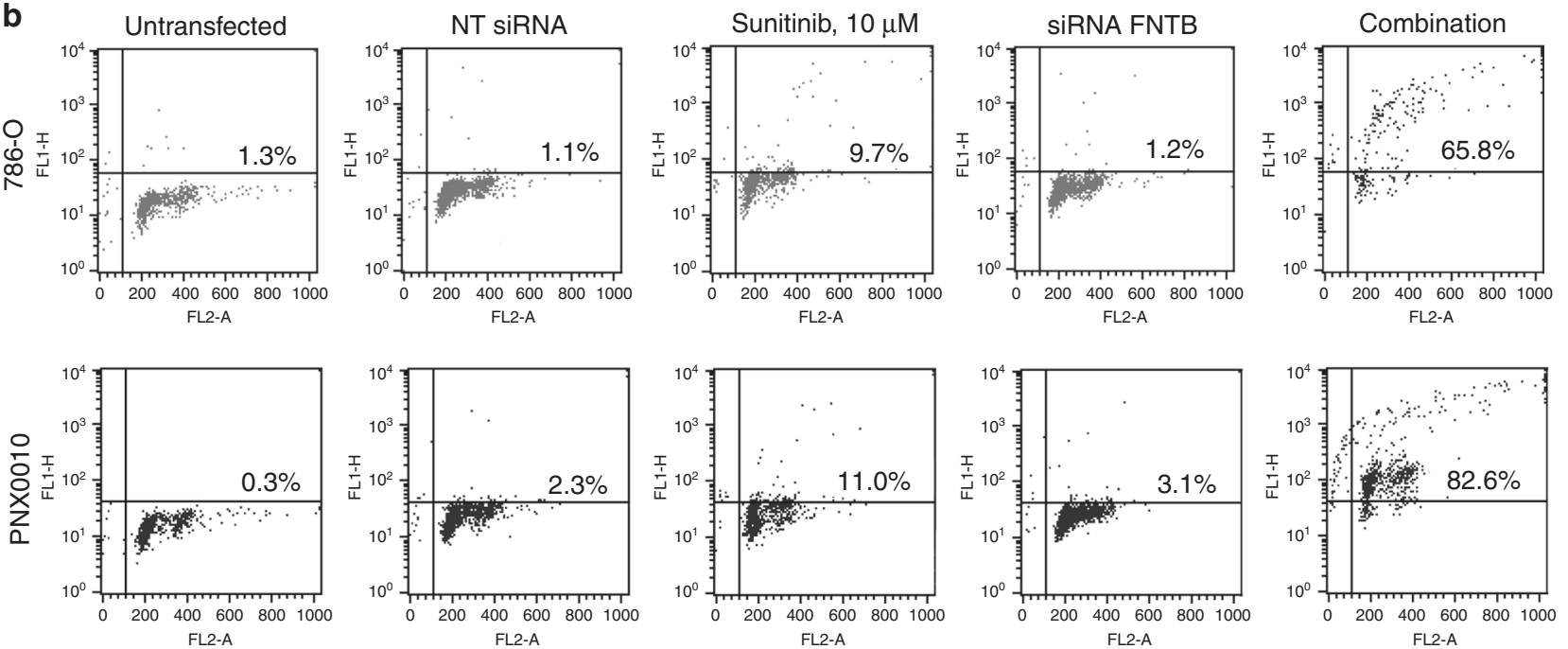

Fig. 2 Validation of the role of farnesyltransferase depletion in resistance to sunitinib in ccRCC. a Western blot analysis of the indicated cCRCC cells transfected with pooled faranesyltransferase $\beta$ subunit (FNTB) siRNA. Negative controls include Non targeting siRNA (NT siRNA) and mock transfection (Mock). b Analysis of sunitinib-mediated apoptosis in the indicated ccRCC cells transfected with FNTB siRNA and Non targeting siRNA. The percentage of apoptotic cells is indicated in the histograms. The representative data of one out of three independent experiments are presented. 
a
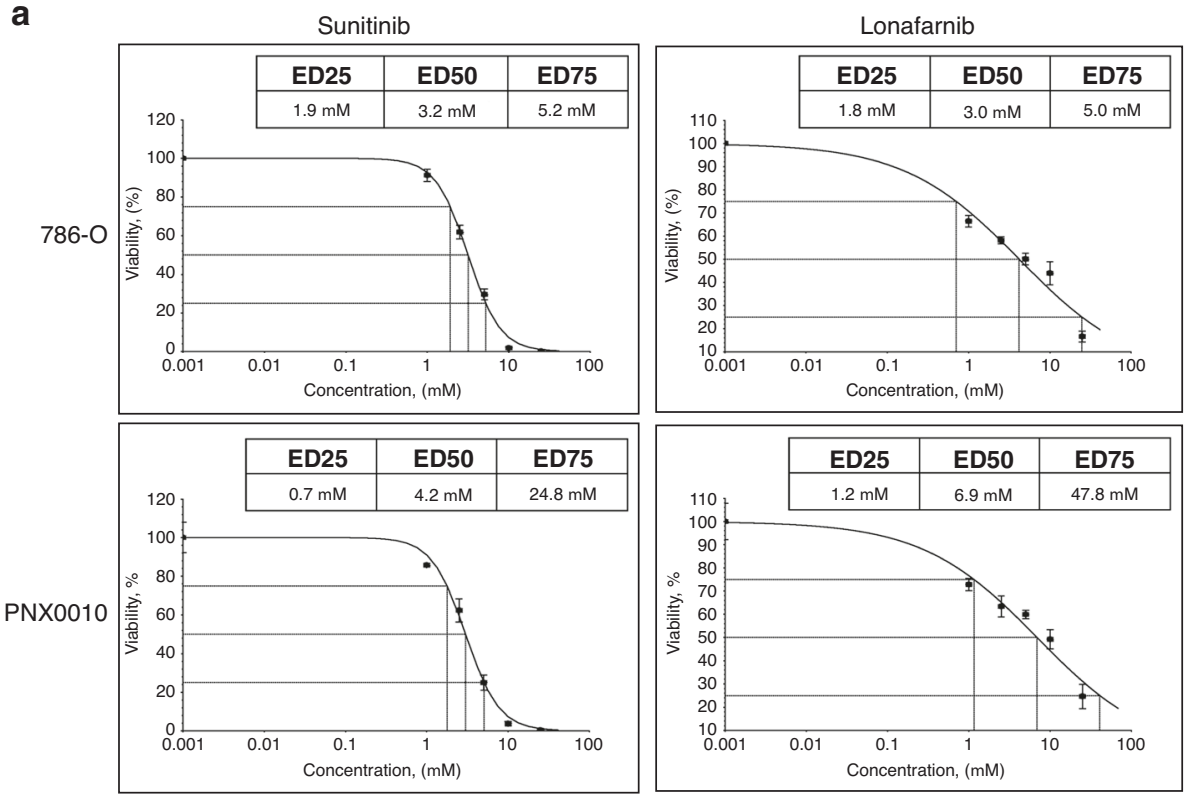

b
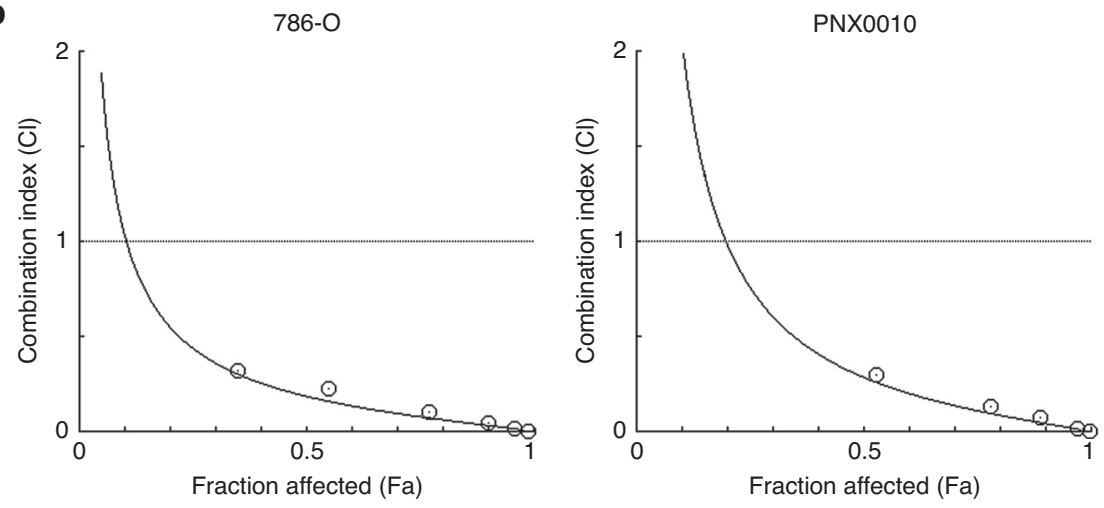

C

\begin{tabular}{|c|c|c|c|c|}
\hline Cell line & Fa $\mathbf{0 . 2 5}$ & Fa $\mathbf{0 . 5}$ & Fa 0.75 & Fa 0.9 \\
\hline $786-\mathrm{O}$ & 0.44 & 0.19 & 0.08 & 0.03 \\
\hline PNX0010 & 0.75 & 0.29 & 0.11 & 0.04 \\
\hline
\end{tabular}

d

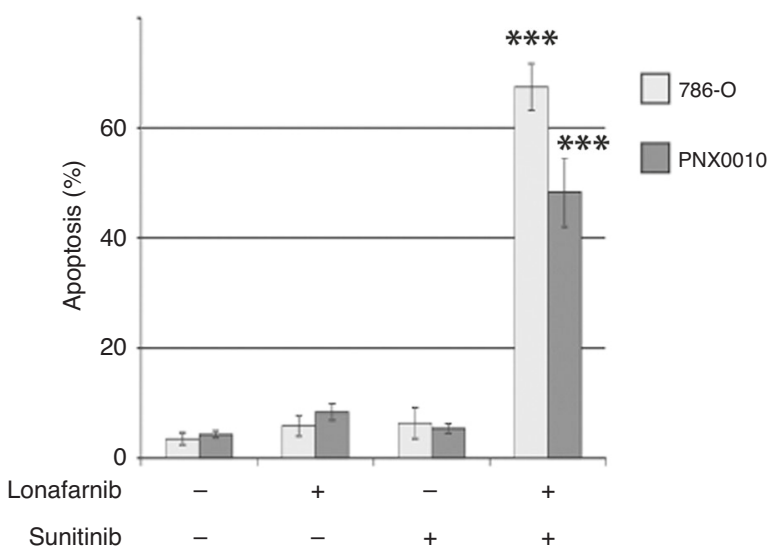

consequently inhibit mTOR signalling. ${ }^{37}$ To address the individual contributions of post-translational prenylation of Rab7a, Rab25 and Rheb proteins, we generated 786-O cells expressing prenylation-incompetent Rab7a, Rab25 and Rheb proteins using CRISPR/Cas9 mediated truncation of C-terminal fragments of those genes containing CAAX motifs. A panel of generated 786-O cellular sub-lines was then treated with sunitinib. As demonstrated in Fig. 5, cells expressing prenylation-incompetent Rheb protein have shown a robust level of apoptosis after sunitinib treatment. These data suggest the critical role of mTORC1 inhibition in FTI- 
Fig. 3 The synergistic effect of combined treatment with sunitinib and lonafarnib on the viability of 786-0 and PNX0010 cells. a Effective doses (EDs) responses of 786-O and PNX0010 cells to sunitinib and lonafarnib. Cells were treated with either sunitinib or lonafarnib for $72 \mathrm{~h}$. Cell viability was examined by CellTiter Blue assay. EDs $(\mu \mathrm{M})$ were calculated using XLfit. $\mathbf{b}$ Combination drug-response curves for the sunitinib plus lonafarnib treatment. 786-O and PNX0010 cells were treated with various combinations of sunitinib and lonafarnib for $72 \mathrm{~h}$. Cell viability was examined by CellTiter Blue assay. $\mathbf{c}$ The combination index $(\mathrm{Cl})$ for the sunitinib plus lonafarnib treatment. The $\mathrm{Cl}$ was calculated using CalcuSyn 2.0 software as described in Materials and Methods. $\mathrm{Cl}>1.3$ : antagonism; $\mathrm{Cl}$ 1.1-1.3: moderate antagonism; $\mathrm{Cl}$ 0.9-1.1: additive effect; $\mathrm{Cl}$ 0.8-0.9: slight synergism; $\mathrm{Cl}$ 0.6-0.8: moderate synergism; $\mathrm{Cl}$ 0.4-0.6: synergism; $\mathrm{Cl} 0.2-0.4$ : strong synergism. d 786-O and PNX0010 cells were treated with sunitinib with or without lonafarnib (both at $10 \mu \mathrm{M}$ ) for $24 \mathrm{~h}$. Apoptosis was examined using APO-BRDU kit followed by flow cytometry analysis. Data are presented as the mean \pm S.D.
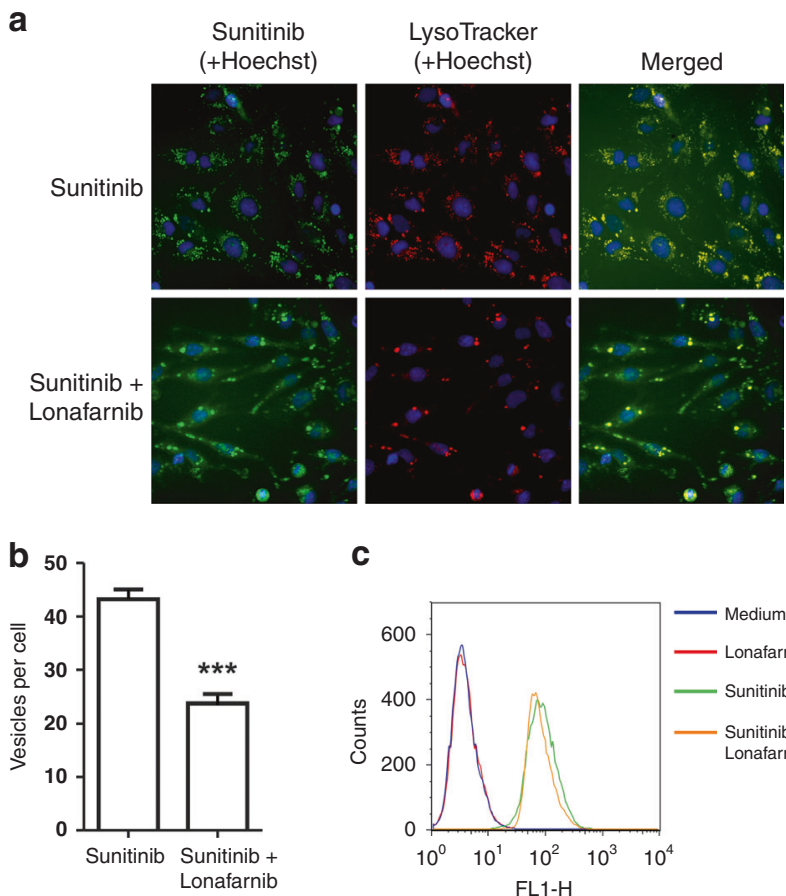

C

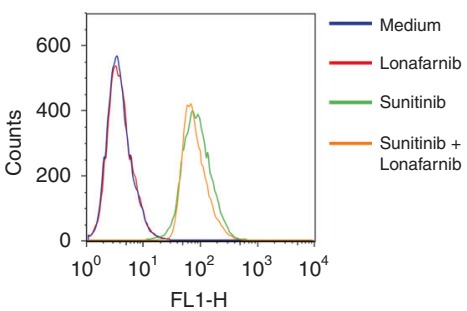

Fig. 4 Lysosomal sequestration of sunitinib. a 786-O cells were treated with sunitinib with or without lonafarnib, stained with Hoechst and Lysotracker Red, and imaged as described in "Methods". b 786-O cells were treated as described in a. The number of sunitinib containing vesicles per cell was calculated as described in Materials and Methods. Data are presented as the mean \pm S.D. ${ }^{* *} P$ $<0.0001$. c The total cellular accumulation of sunitinib was not affected by lonafarnib. 786-O cells were treated as described in a. The samples were run on Becton Dickinson FACScan flow cytometer and analysed using FlowJo software.

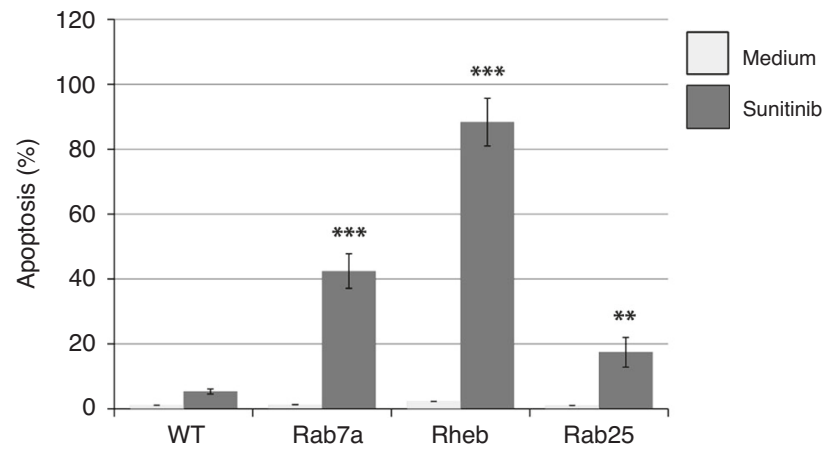

Fig. 5 The role of Rab7a, Rab25 and Rheb prenylation in the resistance to sunitinib in ccRCC cells. 786-O and PNX0010 cells were treated with sunitinib at $10 \mu \mathrm{M}$ for $24 \mathrm{~h}$. Apoptosis was examined using APO-BRDU kit followed by flow cytometry analysis. Data are presented as the mean \pm S.D.

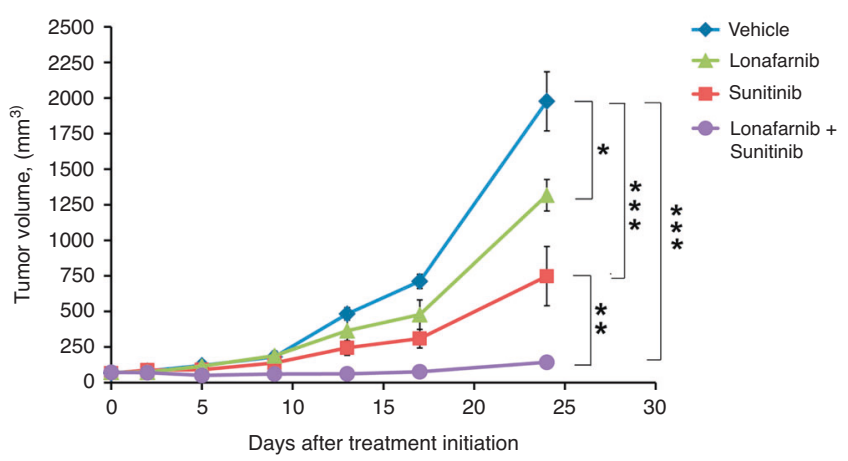

Fig. 6 Treatment with lonafarnib circumvents resistance to sunitinib in a xenograft model of human ccRCC. PNX0010 subcutaneous xenograft tumours were established in 6week-old male C.B17/lcr-scid mice. The mice were treated orally three times per week with: (i) $10 \% 2$-hydroxypropyl- $\beta$-cyclodextrin in PBS (control); (ii) sunitinib ( $40 \mathrm{mg} / \mathrm{kg})$; (iii) lonafarnib (40 mg/kg); (iv) sunitinib and lonafarnib combined. Tumour volumes were calculated as described in "Methods". Values are means $(n=5) \pm$ SD. ${ }^{*} P<$ $0.05 ;{ }^{* *} P<0.001 ;{ }^{* * *} P<0.0001$.

mediated sensitisation of ccRCC cells to sunitinib. 786-O cells expressing prenylation-incompetent Rab7a protein showed reduced level of apoptotic cell death. Expression prenylationincompetent Rab25 protein had mild pro-apoptotic effect on 786-O cells treated with sunitinib (Fig. 5).

In light of our encouraging in vitro data, we next examined the anti-tumour effect of sunitinib in combination with lonafarnib using mice bearing PNXC0010 ccRCC xenograft tumours. As demonstrated in Fig. 6, monotherapy with either sunitinib or lonafarnib showed a moderate decrease in the growth of PNXC0010 xenograft tumours. However, combination treatment with sunitinib or lonafarnib resulted in vastly more impressive inhibition of tumour growth among the experimental groups. These results suggest a readily available clinical strategy to circumvent resistance to sunitinib in ccRCC tumours.

\section{DISCUSSION}

CRISPR-based LOF screening represents the state-of-art tools to identify synthetically lethal combinations for targeted cancer therapy. Our present study using CRISPR/Cas9-based genomewide screen revealed a critical role of FTase as a cellular factor contributing to the resistance of ccRCC cells to sunitinib. The substrate specificity of FTase is determined by the amino acid residues of the CAAX site, in particular the amino acid residue $X .^{38}$ Proteins containing $X$ as methionine or serine exhibit greater affinity for FTase. These are N-Ras proteins containing CysVal-Val-Met, K-Ras4a with Cys-Ile-Ile-Met, K-Ras4b with Cys-Val-IleMet, and H-Ras with Cys-Val-Leu-Ser. ${ }^{39}$ Indeed, FTls were first devised to inhibit Ras, however, alternative prenylation of $\mathrm{K}$ - and $\mathrm{N}$-Ras hinders these drugs' ability to affect Ras oncogenes. ${ }^{40}$ Furthermore, clinical effects of FTIs do not appear to be linked to Ras mutations or inhibition of Ras effectors. ${ }^{41}$ Also, our 
CRISPR/Cas9-based genome-wide screening did not identify Ras and its effectors as central components mediating sunitinib resistance in ccRCC cells. It is therefore likely that other substrates of FTase contribute to the anti-tumour effects of FTase targeted therapeutic agents.

Treatment with TORC1 inhibitors sensitises renal and prostate cancer cells to sunitinib both in vitro and in vivo. ${ }^{9}$ mTORC1 is activated through the direct binding of Rheb, ${ }^{33}$ a GTPase upregulated in transformed cells. ${ }^{34}$ The farnesylation of Rheb is required for its lysosomal membrane localisation and activation of mTORC1 signalling. ${ }^{35,36}$ FTls suppress Rheb farnesylation and consequently inhibit mTOR signalling. ${ }^{37}$ Critically, studies by Meier et al. demonstrate that lonafarnib does not inhibit phosphorylation of ERK or AKT but affects phosphorylation of p70S6K, a downstream target of mTOR signalling. ${ }^{42}$ Therefore, FTIs may potentiate the anti-tumour efficacy of sunitinib, at least in part, through two potential mechanisms: (1) suppression of Rhebdependent mTORC1 activation, and (2) dysregulation of lysosomal sequestration of TKIs. Lysosomal sequestration occurs when a hydrophobic weak base compound enters the lysosome, is protonated in the acid environment, and is unable to cross the membrane. Lysosomal sequestration has been documented for several TKIs including sunitinib, erlotinib, and pazopanib. ${ }^{11,43}$ Interestingly, lysosomal sequestration of sorafenib was observed in hepatocellular carcinoma but not in renal cancer cells. ${ }^{43,44}$ Given that sorafenib does not belong to the same class of hydrophobic weak bases as sunitinib, its lysosomal sequestration could occur via $A B C$ transporter P-glycoprotein (P-gp)-dependent mechanism. ${ }^{45}$ Studies by Colombo et al. demonstrated that treatment with verapamil, a P-gp inhibitor, enhanced the antitumour activity of sorafenib and sunitinib, supporting the role of P-gp in TKIs resistance. ${ }^{44}$ Lysosomal sequestration of hydrophobic weak base therapeutics triggers lysosomal biogenesis. ${ }^{46}$ Enhanced lysosomal biogenesis results in augmented lysosomal drug sequestration and multi-drug cross-resistance. Thus, pharmacological inhibition of FTase may reinstate sensitivity to various TKIs through a common mechanism, i.e. dysregulation of lysosomal drug sequestration via inhibition of TKI-mediated lysosomal biogenesis. Indeed, our findings presented in Fig. 4a, b indicate that treatment with lonafarnib impairs lysosomal biogenesis and thus reduces the amount of sunitinib sequestrated in the lysosomal compartment without affecting the total cellular accumulation of sunitinib.

A number of clinical studies investigating the anti-tumour potential of FTIs led to disappointing results, before it was realised that a biochemical redundancy mechanism allows K-Ras activation by geranylgeranylation (catalysed by GGTase I), which takes over the task of Ras prenylation, when FTase is inhibited. ${ }^{47-49}$ Such redundancy may explain the suboptimal clinical efficacy of FTIs (e.g. tipifarnib) for the treatment of pancreatic $(90 \%$ K-Ras mutations) and lung and colon carcinomas ( 30\% K-Ras mutations). ${ }^{47,50}$ Nevertheless, several Phase 2 trials are currently recruiting patients to study the efficacy of tipifarnib against tumours of various origins (NCT03719690, NCT02383927, NCT03496766, NCT02807272 and NCT02535650).

In summary, our current study presents a promising strategy to identify and validate druggable factors involved in the resistance to targeted therapeutics. Our findings suggest a critical role of FTase-dependent cellular factors in the regulation of sunitinib resistance in cCRCC cells. Future studies are required to precisely elucidate mechanisms underlying synergistic interaction between sunitinib and FTIs.

\section{ACKNOWLEDGEMENTS}

The CRISPR sgRNA library was a gift of Dr. Christoph Seeger (Fox Chase Cancer Center). The authors acknowledge the following Facilities at Fox Chase Cancer Center for their contributions to this work: Flow Cytometry, Laboratory Animal and High Throughput Screening (HTS, Dr. Margret Einarson, supported by R50 CA211479).

\section{AUTHOR CONTRIBUTIONS}

Study concepts/design: P.M. and V.M.K. Data acquisition/data analysis/interpretation: P.M., V.M.K., J.A.S., I.G.S., R.F., V.K., Y.B., and R.G.U. Manuscript preparation: P.M., V.M.K., and I.G.S. Paper review and approval: P.M., Y.B., R.G.U. and V.K.

\section{ADDITIONAL INFORMATION}

Ethics approval and consent to participate All animal studies were performed under approval from the Fox Chase Cancer Center Institutional Animal Care and Use Committee and followed Fox Chase Cancer Center and NIH guidelines. 786-O cell line was obtained from ATCC. PNX0010 cell line was a gift from Dr. Vladimir Khazak (Priaxon Inc.)

Data availability All data are available via the corresponding author.

Competing interests The authors declare no competing interests.

Funding information This work was supported in part by the National Institutes of Health Grants R03 CA216173 and R03 CA246011 (to P.M.); R21 CA223394 (to Y.B. and P.M.); R03 CA212949 and R03 CA235060 (to V.M.K.), the Department of Defense Idea Development Award KC170127 (to V.M.K.), and the NCI Core Grant NCI P30 CA006927 (to Fox Chase Cancer Center).

Supplementary information is available for this paper at https://doi.org/10.1038/ s41416-020-01087-x.

Note This work is published under the standard license to publish agreement. After 12 months the work will become freely available and the license terms will switch to a Creative Commons Attribution 4.0 International (CC BY 4.0).

Publisher's note Springer Nature remains neutral with regard to jurisdictional claims in published maps and institutional affiliations.

\section{REFERENCES}

1. Siegel, R. L., Miller, K. D. \& Jemal, A. Cancer statistics, 2018. CA Cancer J Clin 68, 730 (2018).

2. Shuch, B., Amin, A., Armstrong, A. J., Eble, J. N., Ficarra, V., Lopez-Beltran, A. et al. Understanding pathologic variants of renal cell carcinoma: distilling therapeutic opportunities from biologic complexity. Eur Urol 67, 85-97 (2015).

3. Rini, B. I. Metastatic renal cell carcinoma: many treatment options, one patient. $J$ Clin Oncol 27, 3225-3234 (2009).

4. Rini, B. I. New strategies in kidney cancer: therapeutic advances through understanding the molecular basis of response and resistance. Clin Cancer Res 16, 1348-1354 (2010).

5. Duran, I., Lambea, J., Maroto, P., González-Larriba, J. L., Flores, L., Granados-Principal, S. et al. Resistance to Targeted Therapies in Renal Cancer: The Importance of Changing the Mechanism of Action. Targeted oncology 12, 19-35 (2017).

6. Bridgeman, V. L., Wan, E., Foo, S., Nathan, M. R., Welti, J. C., Frentzas, S. et al. Preclinical Evidence That Trametinib Enhances the Response to Antiangiogenic Tyrosine Kinase Inhibitors in Renal Cell Carcinoma. Mol Cancer Ther 15, 172-183 (2016).

7. Huang, D., Ding, Y., Zhou, M., Rini, B. I., Petillo, D., Qian, C. N. et al. Interleukin-8 mediates resistance to antiangiogenic agent sunitinib in renal cell carcinoma. Cancer Res 70, 1063-1071 (2010).

8. Santoni, M., Amantini, C., Morelli, M. B., Liberati, S., Farfariello, V., Nabissi, M. et al. Pazopanib and sunitinib trigger autophagic and non-autophagic death of bladder tumour cells. Br J Cancer 109, 1040-1050 (2013).

9. Makhov, P. B., Golovine, K., Kutikov, A., Teper, E., Canter, D. J., Simhan, J. et al. Modulation of Akt/mTOR Signaling Overcomes Sunitinib Resistance in Renal and Prostate Cancer Cells. Mol Cancer Ther 11, 1510-1517 (2012).

10. Naito, S., Makhov, P., Astsaturov, I., Golovine, K., Tulin, A., Kutikov, A. et al. LDL cholesterol counteracts the antitumour effect of tyrosine kinase inhibitors against renal cell carcinoma. Br J Cancer 116, 1203-1207 (2017).

11. Gotink, K. J., Broxterman, H. J., Labots, M., de Haas, R. R., Dekker, H., Honeywell, R. $J$. et al. Lysosomal sequestration of sunitinib: a novel mechanism of drug resistance. Clin Cancer Res 17, 7337-7346 (2011).

12. Hillman, G. G., Singh-Gupta, V., Zhang, H., Al-Bashir, A. K., Katkuri, Y., Li, M. et al. Dynamic contrast-enhanced magnetic resonance imaging of vascular changes 
induced by sunitinib in papillary renal cell carcinoma xenograft tumors. Neoplasia 11, 910-920 (2009).

13. Christiansson, L., Söderlund, S., Mangsbo, S., Hjorth-Hansen, H., Höglund, M., Markevärn, B. et al. The tyrosine kinase inhibitors imatinib and dasatinib reduce myeloid suppressor cells and release effector lymphocyte responses. Mol Cancer Ther 14, 1181-1191 (2015).

14. Tamanoi, F., Gau, C. L., Jiang, C., Edamatsu, H. \& Kato-Stankiewicz, J. Protein farnesylation in mammalian cells: effects of farnesyltransferase inhibitors on cancer cells. Cellular and molecular life sciences: CMLS 58, 1636-1649 (2001).

15. Yeganeh, B., Wiechec, E., Ande, S. R., Sharma, P., Moghadam, A. R., Post, M. et al. Targeting the mevalonate cascade as a new therapeutic approach in heart disease, cancer and pulmonary disease. Pharmacol Ther 143, 87-110 (2014).

16. Berndt, N., Hamilton, A. D. \& Sebti, S. M. Targeting protein prenylation for cancer therapy. Nat Rev Cancer 11, 775-791 (2011).

17. Rao, S., Cunningham, D., de Gramont, A., Scheithauer, W., Smakal, M., Humblet, Y. et al. Phase III double-blind placebo-controlled study of farnesyl transferase inhibitor R115777 in patients with refractory advanced colorectal cancer. J Clin Oncol 22, 3950-3957 (2004).

18. Blumenschein, G., Ludwig, C., Thomas, G., Tan, E., Fanucchi, M., Santoro, J. et al. A randomized phase III trial comparing ionafarnib/carboplatin/paclitaxel versus carboplatin/paclitaxel (CP) in chemotherapy-naive patients with advanced or metastatic non-small cell lung cancer (NSCLC). Lung Cancer-J laslc 49, S30-S30 (2005).

19. Harousseau, J. L., Martinelli, G., Jedrzejczak, W. W., Brandwein, J. M., Bordessoule, D., Masszi, T. et al. A randomized phase 3 study of tipifarnib compared with best supportive care, including hydroxyurea, in the treatment of newly diagnosed acute myeloid leukemia in patients 70 years or older. Blood 114, 1166-1173 (2009).

20. Adjei, A. A., Croghan, G. A., Erlichman, C., Marks, R. S., Reid, J. M., Sloan, J. A. et al. A Phase I trial of the farnesyl protein transferase inhibitor R115777 in combination with gemcitabine and cisplatin in patients with advanced cancer. Clin Cancer Res 9, 2520-2526 (2003).

21. Siegel-Lakhai, W. S., Crul, M., Zhang, S., Sparidans, R. W., Pluim, D., Howes, A. et al. Phase I and pharmacological study of the farnesyltransferase inhibitor tipifarnib (Zarnestra, R115777) in combination with gemcitabine and cisplatin in patients with advanced solid tumours. Br J Cancer 93, 1222-1229 (2005).

22. Sparano, J. A., Moulder, S., Kazi, A., Vahdat, L., Li, T., Pellegrino, C. et al. Targeted inhibition of farnesyltransferase in locally advanced breast cancer: a phase I and II trial of tipifarnib plus dose-dense doxorubicin and cyclophosphamide. J Clin Oncol 24, 3013-3018 (2006)

23. Makhov, P., Naito, S., Haifler, M., Kutikov, A., Boumber, Y., Uzzo, R. G. \& Kolenko, V. $M$. The convergent roles of NF-kappaB and ER stress in sunitinib-mediated expression of pro-tumorigenic cytokines and refractory phenotype in renal cell carcinoma. Cell Death Dis 9, 374 (2018).

24. Golovine, K., Makhov, P., Naito, S., Raiyani, H., Tomaszewski, J., Mehrazin, R. et al. Piperlongumine and its analogs down-regulate expression of c-Met in renal cell carcinoma. Cancer Biol Ther 16, 743-749 (2015).

25. Wang, T., Wei, J. J., Sabatini, D. M. \& Lander, E. S. Genetic screens in human cells using the CRISPR-Cas9 system. Science 343, 80-84 (2014).

26. Shalem, O. et al. Genome-scale CRISPR-Cas9 knockout screening in human cells. Science 343, 84-87 (2014)

27. Canter, D., Kutikov, A., Golovine, K., Makhov, P., Simhan, J., Uzzo, R. G. \& Kolenko, V. M. Are all multi-targeted tyrosine kinase inhibitors created equal? An in vitro study of sunitinib and pazopanib in renal cell carcinoma cell lines. The Canadian journal of urology 18, 5819-5825 (2011).

28. Golovine, K., Makhov, P., Uzzo, R. G., Shaw, T., Kunkle, D., Kolenko, V. M. et al. Overexpression of the zinc uptake transporter hZIP1 inhibits nuclear factorkappaB and reduces the malignant potential of prostate cancer cells in vitro and in vivo. Clin Cancer Res 14, 5376-5384 (2008).

29. Chou, T. C. Theoretical basis, experimental design, and computerized simulation of synergism and antagonism in drug combination studies. Pharmacol Rev 58, 621-681 (2006)

30. Morgens, D. W., Deans, R. M., Li, A. \& Bassik, M. C. Systematic comparison of CRISPR/Cas9 and RNAi screens for essential genes. Nature biotechnology 34, 634636 (2016).
31. Kauh, J., Chanel-Vos, C., Escuin, D., Fanucchi, M. P., Harvey, R. D., Saba, N. et al. Farnesyl transferase expression determines clinical response to the docetaxellonafarnib combination in patients with advanced malignancies. Cancer 117, 4049-4059 (2011).

32. Morgillo, F. \& Lee, H. Y. Lonafarnib in cancer therapy. Expert opinion on investigational drugs 15, 709-719 (2006).

33. Parmar, N. \& Tamanoi, F. Rheb G-Proteins and the Activation of mTORC1. The Enzymes 27, 39-56 (2010)

34. Gromov, P. S., Madsen, P., Tomerup, N. \& Celis, J. E. A novel approach for expression cloning of small GTPases: identification, tissue distribution and chromosome mapping of the human homolog of rheb. FEBS Lett 377, 221-226 (1995).

35. Castro, A. F., Rebhun, J. F., Clark, G. J. \& Quilliam, L. A. Rheb binds tuberous sclerosis complex 2 (TSC2) and promotes $\mathrm{S} 6$ kinase activation in a rapamycinand farnesylation-dependent manner. J Biol Chem 278, 32493-32496 (2003).

36. Long, X., Lin, Y., Ortiz-Vega, S., Yonezawa, K. \& Avruch, J. Rheb binds and regulates the mTOR kinase. Curr Biol 15, 702-713 (2005).

37. Basso, A. D., Mirza, A., Liu, G., Long, B. J., Bishop, W. R. \& Kirschmeier, P. The farnesyl transferase inhibitor (FTI) SCH66336 (lonafarnib) inhibits Rheb farnesylation and mTOR signaling. Role in FTI enhancement of taxane and tamoxifen anti-tumor activity. J Biol Chem 280, 31101-31108 (2005).

38. Moores, S. L., Schaber, M. D., Mosser, S. D., Rands, E., O'Hara, M. B., Garsky, V. M. et al. Sequence dependence of protein isoprenylation. J Biol Chem 266, 1460314610 (1991).

39. Klochkov, S. G., Neganova, M. E., Yarla, N. S., Parvathaneni, M., Sharma, B., Tarasov, $\mathrm{V}$. $\mathrm{V}$ et al. Implications of farnesyltransferase and its inhibitors as a promising strategy for cancer therapy. Seminars in cancer biology https://doi.org/10.1016/j. semcancer.2017.10.010 (2017).

40. Braun, T. \& Fenaux, P. Farnesyltransferase inhibitors and their potential role in therapy for myelodysplastic syndromes and acute myeloid leukaemia. British journal of haematology 141, 576-586 (2008).

41. Kurzrock, R., Albitar, M., Cortes, J. E., Estey, E. H., Faderl, S. H., Garcia-Manero, G. et al. Phase II study of R115777, a farnesyl transferase inhibitor, in myelodysplastic syndrome. J Clin Oncol 22, 1287-1292 (2004).

42. Niessner, H., Beck, D., Sinnberg, T., Lasithiotakis, K., Maczey, E., Gogel, J. et al. The farnesyl transferase inhibitor lonafarnib inhibits mTOR signaling and enforces sorafenib-induced apoptosis in melanoma cells. J Invest Dermatol 131, 468-479 (2011).

43. Gotink, K. J., Rovithi, M., de Haas, R. R., Honeywell, R. J., Dekker, H., Poel, D. et al. Cross-resistance to clinically used tyrosine kinase inhibitors sunitinib, sorafenib and pazopanib. Cellular oncology 38, 119-129 (2015).

44. Colombo, F., Trombetta, E., Cetrangolo, P., Maggioni, M., Razini, P., De Santis, F. et al. Giant Lysosomes as a Chemotherapy Resistance Mechanism in Hepatocellular Carcinoma Cells. PloS one 9, e114787 (2014).

45. Azijli, K., Gotink, K. J. \& Verheul, H. M. W. The Potential Role of Lysosomal Sequestration in Sunitinib Resistance of Renal Cell Cancer. Journal of Kidney Cancer and VHL 2, 195-203 (2015).

46. Zhitomirsky, B. \& Assaraf, Y. G. Lysosomal sequestration of hydrophobic weak base chemotherapeutics triggers lysosomal biogenesis and lysosome-dependent cancer multidrug resistance. Oncotarget 6, 1143-1156 (2015).

47. Waller, D. D., Park, J. \& Tsantrizos, Y. S. Inhibition of farnesyl pyrophosphate (FPP) and/or geranylgeranyl pyrophosphate (GGPP) biosynthesis and its implication in the treatment of cancers. Crit Rev Biochem Mol Biol 54, 41-60 (2019).

48. Rowinsky, E. K. Lately, it occurs to me what a long, strange trip it's been for the farnesyltransferase inhibitors. J Clin Oncol 24, 2981-2984 (2006).

49. Yokoyama, K., Zimmerman, K., Scholten, J. \& Gelb, M. H. Differential prenyl pyrophosphate binding to mammalian protein geranylgeranyltransferase-I and protein farnesyltransferase and its consequence on the specificity of protein prenylation. J Biol Chem 272, 3944-3952 (1997).

50. Sparano, J. A., Moulder, S., Kazi, A., Coppola, D., Negassa, A., Vahdat, L. et al. Phase II trial of tipifarnib plus neoadjuvant doxorubicin-cyclophosphamide in patients with clinical stage IIB-IIIC breast cancer. Clin Cancer Res 15, 2942-2948 (2009). 